\title{
Gene Silencing, a Strategy to Induce Virus Resistance in Plants
}

\author{
P. Winterhagen, P. Cobanov, C. Dubois, C. Eisenhauer, Ch. Kehrer, T. Manthey, P. Paschiller, M. Sinn, \\ T. Wetzel, R. Jardak-Jamoussi', G. Krczal, and G.M. Reustle \\ RLP AgroScience GmbH, AIPlanta - Institute for Plant Breeding, Breitenweg 71, 67435 Neustadt a.d. Weinstraße \\ ${ }^{1}$ Institut National de Recherche Scientifique et Technique, Laboratoire de Physiologie Moléculaire de la Vigne, BP 95, \\ Hammam-Lif. 2050, Tunisia \\ Corresondence to: P. Winterhagen, RLP AgroScience GmbH, AlPlanta - Institute for Plant Breeding, Breitenweg 71, \\ 67435 Neustadt a.d. Weinstraße
}

Received: September 5, 2005

The Fanleaf disease, caused by a group of nepoviruses, is a major viral disease in viticulture world-wide. The viral infections dramatically decrease the value of an infested vineyard because of the loss in both the yield and quality of the grapes. Due to a lack of natural genetic resources for viral resistance, suitable for breeding programs, a transgenic approach was chosen to develop rootstocks and cultivars resistant against the most relevant agents of the disease.

Highly conserved sequences of Grapevine Fanleaf Virus (GFLV), Arabis Mosaic Virus (ArMV) and Raspberry Ringspot Virus (RpRSV), were combined with defective interfering (DI)sequences from Tomato Bushy Stunt Virus (TBSV) and/or used to clone directed or inverted-repeat constructs. The strategy allows the induction of sequence specific RNA-silencing by the expression of aberrant and/or dsRNAs, resulting in resistance against viruses with homologous sequences. For proof of concept the constructs were genetically transferred into
Nicotiana benthamiana by Agrobacterium mediated transformation.

Low molecular weight northern blotting showed transgen induced mRNA degradation through PTGS resulting in accumulation of small interfering (si)RNA.

Agrobacterium infiltration with a GFP-virus-sequence containing binary vector demonstrated the efficiently established silencing mechanism in some of the transgenic lines. In these lines, mRNA of GFP was degraded, due to the attached virus specific sequence, resulting in non expression of GFP.

Challenging inoculation with the relevant viruses yielded transgenic tobacco lines showing immunity, recovery, retarded infection and susceptibility.

The experiments could proof that the used constructs can induce virus resistance. However, resistance behaviour between independent transgenic lines as well as within a transgenic line is inconsistent. 